\title{
Advances in Modeling of Polymer Melt Rheology
}

\begin{abstract}
Ronald G. Larson
Dept. of Chemical Engineering, Dept. of Mechanical Engineering, Macromolecular Science and Engineering Program,
\end{abstract} Biomedical Engineering, University of Michigan, Ann Arbor, MI 48109

Qiang Zhou

Dept. of Mechanical Engineering, University of Michigan, Ann Arbor, MI 48109

Sachin Shanbhag

Dept. of Chemical and Biomedical Engineering, FAMU-FSU College of Engineering, Tallahassee, FL 32310

\section{Seung Joon Park}

Dept. of Chemical Engineering and Biotechnology, Korea Polytechnic University, Gyeonggi-do, Siheung, Korea

DOI 10.1002/aic.11064

Published online December 21, 2006 in Wiley InterScience (www.interscience.wiley.com).

Keywords: rheology, polymers, viscoelasticity, constitutive models, diffusion

\section{Introduction}

$\mathrm{T}$ he development of theories to predict the rheological (i.e., flow) properties of densely packed polymers in the melt or solution state is important because such theories might enable rational design of polymer processing methods for shaping polymers into products, and because they can be used in rheological characterization of polymer molecular weight and long-chain branching. These are important topics, given the enormous volume of polymers produced each year (100's of billions of pounds).The seminal work of de Gennes ${ }^{1}$ and Doi and Edwards ${ }^{2-4}$ in the late 1970s established their "tube model" as the standard theory for predicting polymer rheological properties. The "tube" idea arises from the notion that entanglements of a long polymer with its neighbors in a dense melt restrict motion of the polymer to a "tubelike" region - see Figure 1a. Until very recently, the "entanglements" between densely packed long chains that produce a phenomenological "tube" constraining the motion of each chain could not be experimentally imaged or simulated, nor could their existence be rigorously derived from microscopic physics, and so acceptance of the tube model has not come without controversy. In addition, most predictions of the tube model in the early years were hardly better than qualitative.

Correspondence concerning this article should be addressed to R. G. Larson at rlarson@umich.edu.

(C) 2006 American Institute of Chemical Engineers
Still, it was generally recognized early on that, despite its limitations, the Doi-Edwards "tube" provides a plausible ansatz for understanding qualitatively how linear polymers in the entangled state relax, namely, they relax by "reptation" or sliding of the chain along its tube axis, and by retraction within the tube. Moreover, the tube model offered the greatest hope for eventually attaining a quantitative understanding of polymer melt rheology.

In the years since 1978, many efforts have been made to improve upon the Doi-Edwards model. Broadly speaking, the 1980s witnessed a sustained attack on the problems of "primitive-path fluctuations" - that is, changes in the length of the tube due to accordion-like motions of the chain in the tube, ${ }^{5}$ and of "constraint release" — that is, loss of entanglements due to motions of the surrounding chains that define the tube. ${ }^{6,7}$ It was found that as long as the chain "feels" the existence of the tube before the entanglements are lost, one can describe the chain's relaxation as reptation in a tube, where the tube itself is moving through space due to loss, and recreation of entanglements with surrounding mobile chains. This tube movement (now called "constraint release Rouse motion") is especially significant for polydisperse polymers, where the entanglements imposed on long chains by surrounding short chains can relax quite rapidly, and, hence, enhance the mobility of the tube containing the long chain. Finally, in some cases, one can regard the tube diameter to be continuously expanding or "dilating," if the surrounding chains are so much more mobile than the chain in the tube that they act as "solvent" that "dynamically dilutes" the entanglement density. ${ }^{8}$ These ways of incorporating constraint release into tube models have 


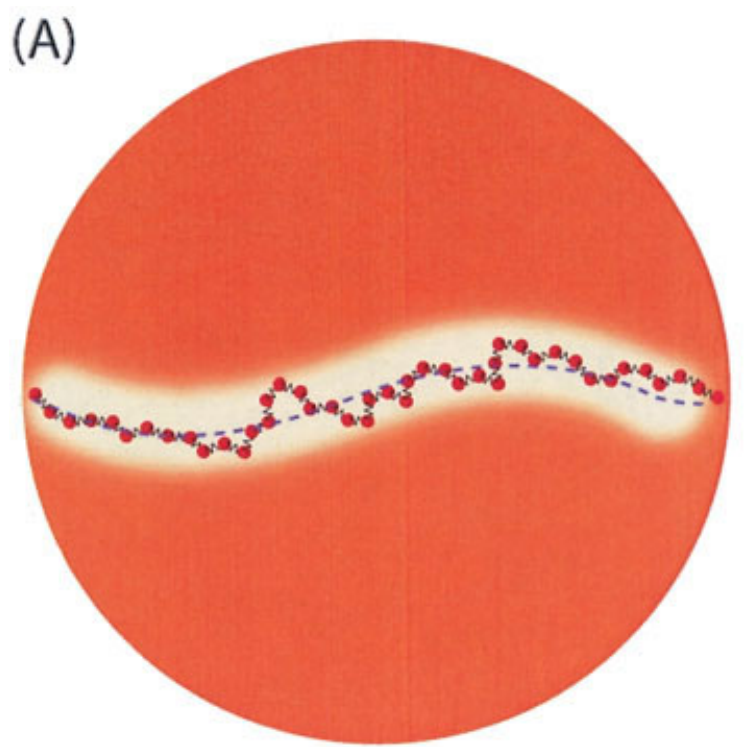

(C)

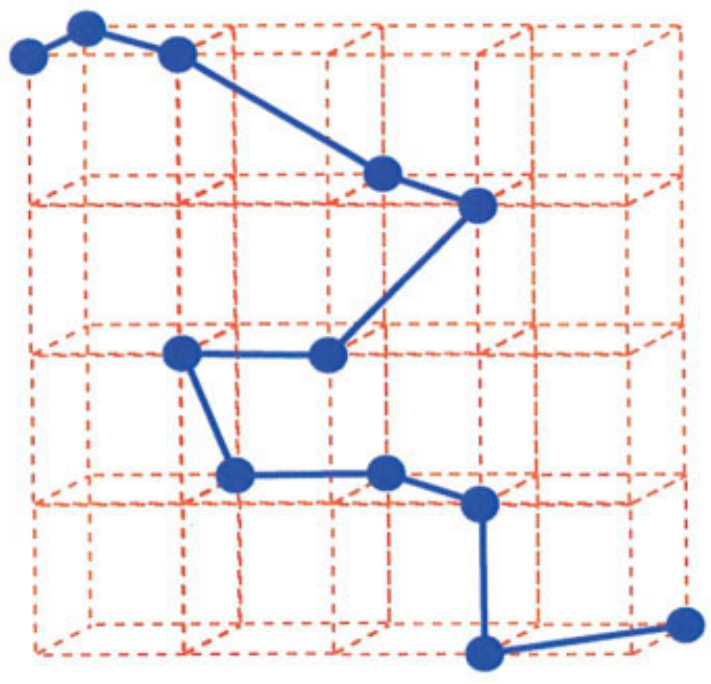

(B)

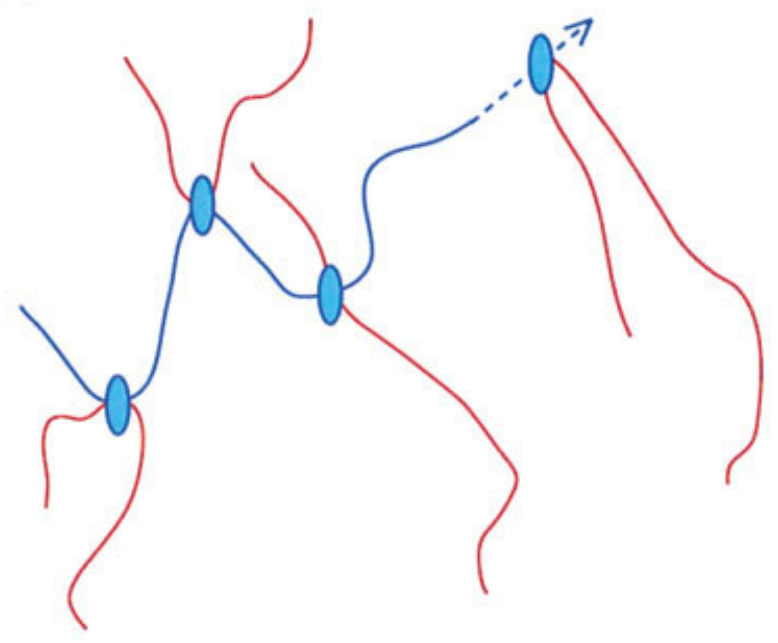

(D)

\section{Polyethylene}

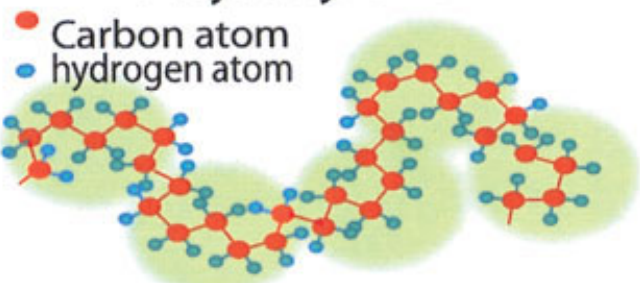

\section{Pearlnecklace Model}

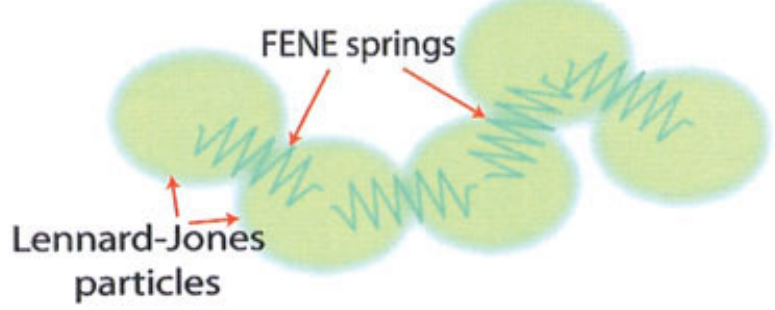

Figure 1. Depictions of (A) the tube model for entangled polymer melts, (B), the slip link model, (C) the bond fluctuation model, and (D) the pearl necklace model.

allowed at least semiquantitative predictions to be made of the linear viscoelastic properties, not only of monodisperse linear and star-shaped polymers, but also of "binary" blends of two different molecular weights of linear polymer, or even mixtures of star and linear polymers. ${ }^{9,10}$

These improvements did little to help overcome major shortcomings in the ability of the Doi-Edwards theory to predict the nonlinear rheology of entangled polymers. However, in the mid1990 's Marrucci ${ }^{11}$ introduced into the tube model a process of "convective constraint release" ("CCR"), that has greatly improved the predictions of the model in fast flows, bringing the tube model to the verge of quantitative accuracy, at least for simple cases of linear monodisperse or bidisperse entangled polymers. ${ }^{10,12}$

While these developments represented huge strides, major gaps in understanding continue to exist, especially in three areas. One is in determining the correct form of the "primitive path potential" governing the fluctuations mentioned previously. This potential represents the statistical unlikelihood of large excursions of the primitive path length from its average length. The potential assumed initially by Kuzuu and Doi ${ }^{13}$ has the form $U / k_{\mathrm{B}} T=v Z\left(L-L_{0}\right)^{2} / L_{0}$, where $v=3 / 2$ is a constant prefactor, $L$ is the fluctuating primitive path length, and $L_{0}$ is the average value of $L$. The distribution of primitive paths is then given by $\psi(L) \propto \exp \left(-U(L) / k_{\mathrm{B}} T\right)$. The Kuzuu-Doi form of the potential has never been rigorously justified. This primitive-path potential is especially important for relaxation of branched polymers, which cannot relax by reptation because of the anchoring effect of the branch point. A second gap is in the details of the "dilution" process, typically subsumed into a "dilution exponent" $\alpha$ that determines how rapidly relaxation of surrounding molecules widens the tube. A third problem is 
to determine how rapidly branch points move in response to relaxation of the attached branches. Besides these problems with the physics of polymer relaxation, there is the logistical problem of incorporating all relevant phenomena into equations or algorithms that can predict accurately the linear and/or nonlinear rheology of commercial melts with broad distributions of molecular weight and of branch structure.

Increasingly, progress in all these areas has come from computational methods. As we will describe later, fine-grained computations are now beginning to allow us to show in detail how the elusive "entanglements," "tubes" and "primitive paths" assumed by the tube model arise from the local polymer structure and dynamics. In addition, algorithmic advances are enabling tubemodel computations to cope with polydispersity by tracking the simultaneous relaxations of large ensembles of molecules. ${ }^{14}$

In the following, we briefly review microscopic models, namely the pearl necklace, lattice, and slip link models — see Figure $1 \mathrm{~b}-\mathrm{d}$, that have shown the most promise for exposing the detailed physics underlying the tube model, and correcting its deficiencies. Then, we discuss the extent to which the tube model has been improved and exploited in recent years to predict the linear and nonlinear rheology of the polydisperse linear and branched melts used commercially.

\section{Pearl Necklace Simulations}

Kremer and Grest ${ }^{15,16}$ analyzed the microscopic basis of entanglement dynamics by applying molecular dynamics (MD) simulations to the "pearl-necklace" model (see Figure 1d). This model represents a polymer molecule as a string of repulsive Lennard-Jones beads linked together by the finitely-extensible nonlinear elastic (FENE) springs that are short and stiff enough to keep beads on surrounding chains from passing between them. Entanglements between chains, are, therefore, enforced, and each bead is a coarse-grained monomer corresponding roughly to a "Kuhn" random-walk step length. MD simulations of the pearl-necklace chain can predict some rheological properties of entangled polymer melts that would be computationally too demanding to capture in atomistic simulations. While the resolution of the pearl necklace model is fine enough that chain configurations are realistically captured, simulating densely entangled chains (more than around 10 entanglements) out to their longest relaxation time is only possible with months-long simulations on supercomputers. Hence, such simulations focus on mildly entangled systems or shorter-time behavior of long chains. Simulations of slow dynamics of densely entangled linear or branched chains require models that are less detailed, such as the lattice or the slip-link models discussed in the next sections.

Over the last couple of decades, MD pearl-necklace simulations have progressed from verifying that the basic reptation picture is qualitatively accurate, to more recent work that attempts to resolve the microscopic basis of entanglement dynamics. In the early work, the signature of reptation along a "tube" was found in the transition of the mean-squared monomer diffusion from $\mathrm{t}^{1 / 2}$ scaling at early times $\mathrm{t}$ corresponding to an unentangled three-dimensional (3-D) Rouse regime, where beads have not moved far enough to "feel" the tube, to $t^{1 / 4}$ scaling at longer times where the bead motion is dominated by 1-D Rouse diffusion along the tube. ${ }^{15}$ This transition had been predicted by the tube theory. ${ }^{17}$ The transition of the zero-shear viscosity as a function of molecular weight from a linear regime to a 3.4-power-law regime, which is taken to be evidence of the entanglement effects, was also captured in MD simulations by Kröger and Hess. ${ }^{18}$

More recently, in a major breakthrough, Everaers et al. ${ }^{19}$ identified primitive paths of the tubes directly through a self-consistent "cooling" procedure in which all chains are "tightened" by simultaneously removing their slack without allowing them to pass through each other. This process yields a network of taut, interlacing, primitive paths, such as that shown on the cover of this journal. Since the primitive path is the key theoretical entity underlying all tube theories, an ability to determine it directly from the microscopic molecular configurations means that, for the first time, coarse-grained quantities related to the tube can be derived from atomic-scale physics. For example, Everaers et al. showed that the average primitive path length derived from their method yields an estimate of $N_{\mathrm{e}}$, the number of beads (or "Kuhn steps") per entanglement, that is consistent with the estimate derived from the plateau modulus (a readily measured rheological property) using the tube model. Thus, the work of Everaers et al., published in Science, triggered intensive efforts by multiple groups to analyze the primitive paths from microscopic simulations.

While it predicts a reasonable value for the average primitive-path length, the method of Everaers et al. yields a narrower distribution of primitive-path lengths than the Doi-Kuzuu ${ }^{13}$ distribution. ${ }^{20}$ That is, with the method of Everaers et al., the distribution is characterized by a potential $U / k_{\mathrm{B}} T=v Z\left(L-L_{0}\right)^{2} / L_{0}$ in which the prefactor $v$ is around 3.0, twice the Doi-Kuzuu value of 1.5. Zhou and $\operatorname{Larson}^{20}$ showed that Everaers' cooling method is equivalent to minimizing a total quadratic spring energy of the polymer sample. Switching to linear potential during the cooling procedure, which corresponds to minimizing the total length of primitive paths, yields a distribution with a broader width (corresponding to $v=1.5$ ), in agreement with the Doi-Kuzuu distribution. The values of $N_{\mathrm{e}}$ estimated from the two methods are surprisingly almost the same, however. Thus, it appears that a reasonable method of identifying the primitive paths, namely minimizing total primitive path length by slow "cooling," subject to fixed chain ends and noncrossability of chains, confirms the correctness of the original primitive path potential assumed by the tube model.

Although it represents a conceptual breakthrough, the "cooling" method of identifying primitive paths is not free from difficulties. First, the primitive path has a finite tension that deforms the entanglement network. Second, the primitive path has a finite thickness, addressed by Tzoumanekas and Theodorou, ${ }^{21}$ which leads to overly long primitive paths due to the extra length one path takes to wrap around another. Lastly, cooling methods are very computation-intensive. Fortunately, Kröger's shortest path method, ${ }^{22}$ based on pure geometry, is much faster, especially if coupled with the fastmelt equilibration method known "double-bridging Monte Carlo," allowing Kröger and coworkers to obtain much better statistics of primitive paths, and study a wider parameter range $^{23}$ than possible with other methods. They found that the relative width of the distribution of the primitive path length is chain-length dependent, that is, the longer the polymer chain, the wider the distribution of primitive path lengths, with the longest chain lengths (with 16 entanglements) yielding a primitive path width corresponding to around $v=1.1$. 


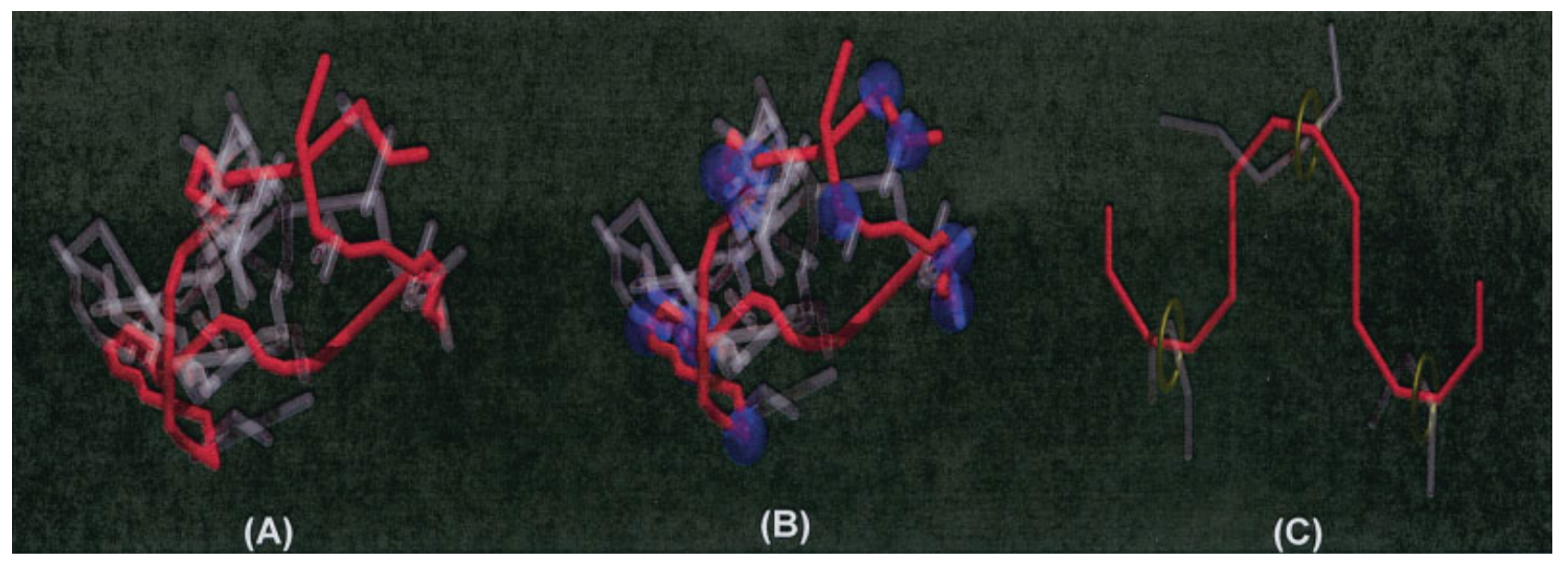

Figure 2. (A) After applying the primitive-path identification algorithm to a system of chains equilibrated using the lattice bond-fluctuation model, a particular primitive chain (red) constrained by matrix chains (transparent white) is shown; (B) using geometrical arguments, positions of topological constraints or entanglement points (blue spheres) encountered by the primitive chain can be identified. There appears to be a one-to-one correspondence between these entanglement points and constraining matrix chains and (C) one could in principle derive a slip link model from a coarse-grained representation of the system depicted in (B).

Each slip link (golden ring) constrains two chains, although allowing them to slide through it. Note that, for clarity, the number of slip links in $C$ is fewer than the number of entanglement points identified in $B$.

This chain-length dependence is quite close to that predicted theoretically by Schieber. ${ }^{24}$

Which of the various definitions for the "primitive path" is closest to the ideal "primitive path" conjectured by the tube model is still an open question. In the near future, we hope to compare the primitive paths from different methods and determine either that all reasonable methods of minimizing overall primitive path length give the same distribution, or, if not, identify the most nearly "correct" method for determining primitive paths.

\section{Lattice Models}

Polymer chains on a lattice have for many years been used to explain static properties such as the "random walk" or "swollen" configurations of polymer chains in melts and dilute solutions, respectively. The bond-fluctuation model (BFM), ${ }^{25,26}$ depicted in Figure 1c, represents the state-of-the-art in lattice models, and is more realistic than previous lattice models. The BFM approximates continuum MD simulations while preserving the computational efficiency associated with integer operations. In the BFM, the length of the "coarse-grained" bond connecting consecutive "effective monomers" varies somewhat to allow bond angles to vary over a wider range than in a conventional cubic lattice. By restricting the number of allowable bond vectors connecting adjacent monomers to 108 , the BFM can enforce chain noncrossability, allowing reptationlike behavior to be modeled. Shanbhag and Larson ${ }^{27}$ adapted the primitive path identification algorithm of Everaers et al. to Shaffer's version of the $\mathrm{BFM}^{26}$ to obtain the primitive paths via a "cooling" method (see the section titled Pearl Necklace Simulations). Figure 2 a depicts a particular primitive chain surrounded by other "matrix" primitive chains. These lattice simulations, with subsequent corroboration by off-lattice molecular dynamics simulations (discussed in the section titled Pearl Necklace Similations), support the originally proposed quadratic form of the fluctuation potential, in good agreement with the Doi-Kuzuu distribution (with $v=1.5$ ), although for longer chains (with $Z=16$ entanglements) the distribution was a little wider (corresponding to $v=1.36$ ).

The motion of a polymer chain residing in a concentrated solution or melt is substantially accelerated due to the simultaneous motion of surrounding chains, and the bare-tube fluctuation potential is softened or "dynamically diluted". ${ }^{8}$ Whether this dynamically diluted fluctuation potential can be derived using lattice or molecular-dynamics simulations remains an open question. However, by appealing to a simple geometrical argument within the framework of the BFM, Shanbhag and Larson $^{28}$ constructed an algorithm to locate the positions of topological constraints or "entanglements" encountered by the primitive paths. Figure $2 \mathrm{~b}$ shows the locations of the topological constraints encountered by the primitive path depicted in Figure 2a due to the surrounding chains. Counting the number of these "entanglement" points removed when single chains were removed from the lattice yields an estimate that the value of the dilution exponent $\alpha$ is close to unity. This implies that, on average, only two chains participate in the creation of each entanglement, which is consistent with the idea that entanglements arise due to binary interactions between chains, and is a core assumption of slip link models described in the next section.

\section{Slip Link Models}

In order of increasing degree of coarse-graining, slip link models follow after first pearl necklace models and lattice models, both of which are resolved at the level of the Kuhn step length. In slip link models, molecular details at the monomer or Kuhn step level are obscured; however, among microscopic simulations, slip links models are of the greatest relevance to rheology, precisely because coarse-graining allows timescales of the order of the longest-polymer relaxation time to be accessed, which is not possible for finer grained models, except 


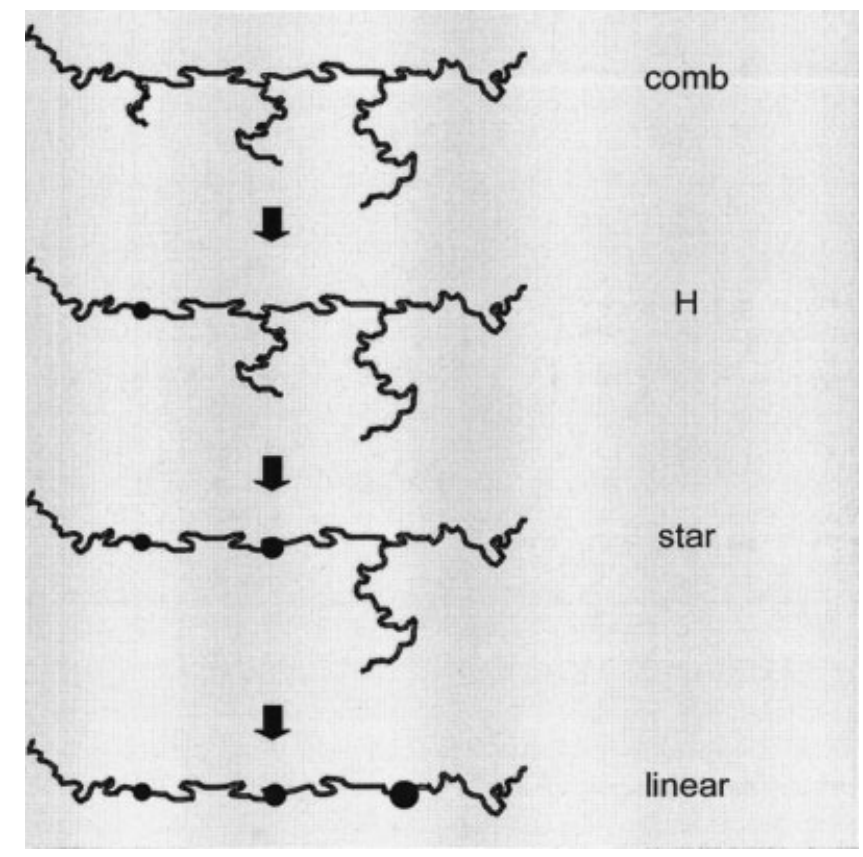

Figure 3. Conceptualization of algorithm for computing hierarchical relaxation of a comb-branched polymer. Note that on relaxation, the comb molecule effectively becomes an " $\mathrm{H}$," molecule, then a star, and then a linear molecule, as the arms progressively relax, and at long times behave as frictional sites. ${ }^{34}$

for rather short chains that are only weakly entangled. In slip link models, a polymer melt is modeled in a manner analogous to a crosslinked network, where instead of being permanent as crosslinks are, the slip links are temporary and allow monomers on each chain constrained by them to slide through the slip link constraint. As shown in Figure 1b and 2c, each slip link constrains two chains, and is a proxy for an entanglement. Chains wiggle around via Brownian motion, and the slip links can themselves be either fixed in space or mobile, depending on how they are modeled. When either of the constrained chains slithers out of a slip link, the slip link is destroyed. Similarly, the end of one chain (the "test" chain) can hop toward another chain (the "matrix" chain), and, thereby, create a new slip link or entanglement. Microscopic information, such as the ordering, spatial location, and age of surviving slip links can be related mathematically to macroscopic properties, such as stress and dielectric relaxation functions.

Since the first slip link model of Hua and Schieber $^{29}$ was introduced, several other models differing in the level of resolution and algorithmic detail have emerged. The simplest of these is the "virtual space" slip link model of Shanbhag et al., ${ }^{30}$ which has been helpful in understanding the conditions under which the dynamic dilution theory for branched polymers is valid. Quantitative predictions of the nonlinear rheology of linear polymers, including strain hardening in extensional flows in polystyrene samples, have been made using the slip link model of Doi and Takimoto. ${ }^{31}$ While rheology has been the primary focus of slip link models, the model of Likhtman ${ }^{32}$ has been used to describe complementary experimental data on monodisperse-linear polymers, such as neutron spin-echo, and diffusion, simultaneously with rheology. Currently one of the most detailed slip link models is "NAPLES," developed by Masubuchi et al., ${ }^{33}$ which is essentially a Brownian-dynamics simulation of a network of primitive paths. The motion of the slip links is governed through a force balance on entanglement nodes, while the sliding motion of monomers along the chain contour is regulated by the distribution of tension in the chain. Extensions of NAPLES have been used to study branched polymers, nonlinear rheology, phase separation dynamics in polymer blends, and to address important questions regarding entanglement spacing and the plateau modulus. To date, neither finer-grained pearl necklace nor lattice simulations have been directly mapped onto a slip link model, using the finer-grained model to identify explicitly the spatial locations of the entanglement points that are modeled as slip links. Such an exercise could help determine which of the various slip link models proposed so far is the most realistic.

\section{Tube Model - Linear Viscoelasticity}

The ideas of reptation, primitive path fluctuations, and constraint release by "constraint release Rouse motion" and dynamic dilution are able, when combined, to predict the linear rheological properties of linear, star-branched, and more complex branched structures, such as " $\mathrm{H}$ " and "comb" structures (see Figure 3 ) that are specially made to be nearly monodisperse. To describe commercial branched polymer melts, these molecular theories must be generalized so that they can be applied to polymers that are not only polydisperse in molecular weight,but also in branch length and branch placement. Larson ${ }^{14}$ developed an algorithm that generalized the Milner-McLeish theories to allow prediction of the relaxation of general mixtures of branched polymers. He called this the "hierarchical model" because it is based on hierarchical relaxation in branched polymers, in which relaxation starts at the tips of arms and proceeds inwards. A key aspect of this approach is that it represents polydisperse melts by a large set (up to 10,000 or so) of molecular structures that are generated stochastically on the computer, and are used to represent the range of molecular weights and branching structures of polymers in the real melt. The algorithm then tracks the relaxation of each of these molecules and how this contributes to the dynamic dilution that affects the relaxation of the other molecules in the ensemble. Park et al. ${ }^{34}$ modified this algorithm to allow for more quantitative predictions, in part by including the "early time fluctuations." Figure 3 illustrates how the model treats the hierarchical relaxation of a comb-branched polymer, which is composed of arms and backbone segments. Park et al. ${ }^{34}$ showed that the modified hierarchical model successfully predicts linear viscoelastic data for various model branched polymers and branched/linear mixtures, and thus might be a promising algorithm for predicting the relaxation of general mixtures of branched polymers.

Since the hierarchical model is quite general, it could be applied to commercial long-chain branched polymers, such as single-site metallocene polyethylenes, as long as the branching density is small (less than an average of 1 branch per 10 molecules), so that branches on branches can be ignored. The development of single-site "constrained geometry" metallocene catalysts has made it possible to introduce controlled levels of long-chain branching (LCB) into commercial linear polyethylenes. Controlled levels of LCB in metalocene-catalyzed polyethylenes (mPE's) now allow one to carry out sys- 
tematic studies of the effect of LCB on the rheological properties of polyethylenes keeping the MW and MWD almost unchanged, as has been done by Wood-Adams and coworkers. ${ }^{35}$ From a single-site model, a complete statistical description of the branching characteristics of metallocenecatalyzed high-density polyethylenes (m-HDPE) can be generated, including the distribution of branching locations, and lengths using only two independent parameters that control all characteristics of the distribution. ${ }^{36}$ Using the statistical model for these polyethylenes, Park and Larson ${ }^{34}$ predicted accurately the linear viscoelasticity of m-HDPE having different degrees of the long-chain branching using the hierarchical model with the parameters determined a priori.

The hierarchical model developed by Park and Larson did not permit consideration of branches on branches, which are produced at appreciable concentration $(>1 \%)$ by constrained geometry catalysts once the branching density exceeds around 0.1 long branches per molecule. However, recently Das et al. ${ }^{37}$ extended the approach to allow prediction of polymers with branch-on-branch structures. Their model predictions are in quantitative agreement with linear rheological data for single-site $\mathrm{m}$-HDPE over a wide range of branching densities, up to 0.5 long branches per molecule. While the experimental data for LCB m-HDPE are not extensive enough to test them completely, if these models prove to be robust, then, when combined with other characterization techniques such as gel permeation chromatography, they could be used to infer branching levels from measurements of the linear viscoelastic properties of LCB mHDPE.

\section{Tube Model - Nonlinear Viscoelasticity}

The modeling of nonlinear viscoelasticity of polymer melts, especially melts that are polydisperse and/or have long-chain branching, is vastly more complicated than is the prediction of linear-viscoelastic properties. While one can characterize fully the linear viscoelastic response of a material by its relaxation after a small step-shear strain, nonlinear viscoelasticity is sensitive to changes in both time history and flow type. In addition, theories for nonlinear viscoelasticity are difficult to test rigorously, since accurate rheological data are difficult to obtain in the strongly nonlinear regime.

As a result of these difficulties, modeling of nonlinear rheology, and testing of these models, has for the most part been limited to concentrated solutions, and to a lesser extent, melts of linear (i.e., unbranched) polymers. As alluded to earlier, accurate predictions of nonlinear rheology using the tube model requires incorporation of "convective constraint release" (CCR), which is the release of entanglement constraints by convective flow. ${ }^{11}$ The inclusion of CCR has yielded constitutive equations that qualitatively match the shear-flow rheology of monodisperse and bidisperse linear polymers. ${ }^{38} \mathrm{~A}$ recent milestone is the successful prediction of both birefringence patterns and neutron scattering patterns of a nearly monodisperse polymer melt flowing through a contraction flow, using a nonlinear constitutive equation, based on the tube model that includes reptation, chain stretch, and CCR. ${ }^{39}$ The development and testing of constitutive models, based on the tube model for polydisperse linear polymers is a top priority, and there is likely to be significant progress along these lines in the near future.

For long-chain branched polymers, the only available nonlinear constitutive equations based on the tube model apply only to ideal molecular architectures, and even then are only qualitative. $^{40}$ There are indications that stochastic models, such as slip link models, might be able to cope with the complexity of the nonlinear rheology of polydisperse polymers, ${ }^{31}$ and possibly eventually with long-chain branching as well.

\section{Summary and Prognosis}

These are exciting times in the field of polymer rheology. Computational power is finally reaching the point that entanglement interactions that occur on the microsecond timescale or longer can be directly tracked by fine-grained simulations resolved at the level of the monomer or "Kuhn step length." These simulations have generally supported the notion of a tube, and the traditionally accepted mechanisms of relaxation in the tube, and have allowed the heretofore elusive entities assumed by the tube model — that is, the primitive-path length, tube diameter, fluctuation potential, and even individual "entanglement points" - to be determined directly from the fine-grained model. Fine-grained simulations are also beginning to suggest corrections or reinterpretations that might be needed in the theory. To bring this promising work to consummation, agreed-upon methods of obtaining primitive paths must be established, or it must be shown that results are insensitive to them. Another important remaining task for finegrained simulations is the analysis of branch-point motion.

Increased computational power has also permitted the successful application of the coarse-grained tube or slip link models to prediction of the linear rheology of very complex polydisperse melts, or melts with irregular long-chain branching. This success has come at a good time, as novel commercial catalysts that provide unprecedented control over molecular weight and branching distributions can only be exploited fully if theory is developed that will allow determination of the structures that give the best rheological properties. Successful initial work predicting the linear rheology of commercial-branched polymers must be followed up with predictions of rheological properties of commercial melts across a range of molecular weights and branching distributions to determine the robustness of the methods.

Finally, while progress has been made analyzing and predicting the nonlinear rheological properties of the simplest linear-polymer chains that are monodisperse or bidisperse, predictions of nonlinear properties for more complex melts, including commercial melts, continues to lag. Achieving predictions of nonlinear properties with accuracy and versatility comparable to that already achieved for linear properties will take considerable effort. However, it is a much needed effort, since it is the nonlinear rheology that controls processing. Hence, accurate and versatile nonlinear rheological models must be included in the suite of modeling tools that will ultimately allow polymerization reactions to be designed to produce polymers with optimal processing characteristics.

\section{Literature Cited}

1. de Gennes PG. Reptation of a polymer chain in the presence of fixed obstacles. J. Chem Phys. 1971;55:572-579.

2. Doi M, Edwards SF. Dynamics of concentrated polymer systems. Part 1 - Brownian motion in the equilibrium state. J of the Chemical Society. Faraday Transations II. 1978;74:1789-1801. 
3. Doi M, Edwards SF. Dynamics of concentrated polymer systems. Part 2 - Molecular motion under flow. $J$ of the Chemical Society. Faraday Transactions II. 1978;74: 1802-1817.

4. Doi M, Edwards SF. Dynamics of concentrated polymer systems. Part 3 - The constitutive equation. $J$ of the Chemical Society. Faraday Transactions II. 1978;74:1818-1832.

5. Doi M. Explanation for the 3.4 power law of viscosity of polymeric liquids on the basis of the tube model. $J$ of Polymer Sci. Polymer Physics Ed. 1983;21:667-684.

6. Viovy JL, Rubinstein M, Colby RH. Constraint release in polymer melts: Tube reorganization versus tube dilution. Macromolecules. 1991;24:3587-3596.

7. Watanabe H. Viscoelasticity and dynamics of entangled polymers. Progress in Polymer Science. 1999;24:1253-1403.

8. Ball RC, McLeish TCB. Dynamic dilution and the viscosity of star polymer melts. Macromolecules. 1989;22: 1911-1913.

9. McLeish TCB. Tube theory of entangled polymer dynamics. Advances in Physics. 2002;51:1379-1527.

10. Milner ST, McLeish TCB, Young RN, Hakiki A, Johnson JM. Dynamic dilution, constraint-release, and star-linear blends. Macromolecules. 1998;31:9345-9353.

11. Marrucci G. Dynamics of entanglements: a nonlinear model consistent with the Cox-Merz rule. J of Non-Newtionian Fluid Mechanics. 1996;62:279-289.

12. Dealy J, Larson RG. Structure and rheology of molten polymers. From structure to flow behavior and back again. Munich: Hanser; 2006.

13. Doi M, Kuzuu NY. Rheology of star polymers in concentrated-solutions and melts. $J$ of Polymer Sci C-Polym Letts. 1980;18:775-780.

14. Larson RG. Combinatorial rheology of branched polymer melts. Macromolecules 2001;34:4556-4571.

15. Kremer K, Grest GS, Carmesin I. Crossover from Rouse to reptation dynamics - a molecular-dynamics simulation. Phys Rev Letts. 1988;61:566-569.

16. Kremer K, Grest GS. Dynamics of entangled linear polymer melts - a molecular-dynamics simulation. $J$ of Chem Phys. 1990;92:5057-5086.

17. Doi M, Edwards SF. The theory of polymer dynamics. Oxford: Oxford University Press; 1988.

18. Kröger M, Hess S. Rheological evidence for a dynamical crossover in polymer melts via nonequilibrium molecular dynamics. Phys Rev Letts. 2000;85:1128-1131.

19. Everaers R, Sukumaran SK, Grest GS, Svaneborg C, Sivasubramanian A, Kremer K. Rheology and microscopic topology of entangled polymeric liquids. Science. 303:823-826.

20. Zhou Q, Larson RG. Primitive path identification and statistics in molecular dynamics simulations of entangled polymer melts. Macromolecules. 2005;38:5761-5765.

21. Tzoumanekas C, Theodorou DN. Topological analysis of linear polymer melts: a statistical approach. Macromolecules. 2006;39:4592-4604.

22. Kröger M. Shortest multiple disconnected path for the analysis of entanglements in two- and three-dimensional polymeric systems. Comp Phys Communications. 2005;168:209-232.

23. Foteinopoulou K, Karayiannis NC, Mavrantzas VG, Kröger M. Primitive path identification and entanglement statistics in polymer melts: Results from direct topological analysis on atomistic polyethylene models. Macromolecules. 2006;39:4207-4216.

24. Schieber JD. Fluctuations in entanglements of polymer liquids. J of Chem Phys. 2003;118:5162-5166.

25. Carmesin I, Kremer K. The bond-fluctuation method - a new effective algorithm for the dynamics of polymers in all spatial dimensions. Macromolecules. 1988;21:2819-2823.

26. Shaffer JS. Effects of chain topology on polymer dynamics - bulk melts. Journal of Chemical Physics. 1994;101:4205-4213.

27. Shanbhag S, Larson RG. Chain retraction potential in a fixed entanglement network. Phys Rev Letts. 2005;94:076001.

28. Shanbhag S, Larson RG. Identification of topological constraints in entangled polymer melts using the bondfluctuation model. Macromolecules. 2006;39:2413-2417.

29. Hua CC, Schieber JD. Segment connectivity, chain-length breathing, segmental stretch, and constraint release in reptation models. I. Theory and single-step strain predictions. J of Chem Phys. 1998;109:10018-10027.

30. Shanbhag S, Larson RG, Takimoto J, Doi M. Deviations from dynamic dilution in the terminal relaxation of star polymers. Phys Rev Letts. 2001;87:195502.

31. Doi M, Takimoto J-I. Molecular modeling of entanglement. Philosophical Transactions of the Royal Society London A. 2003;361:641-652.

32. Likhtman AE. Single-chain slip-link model of entangled polymers: simultaneous description of neutron spin-echo, rheology, and diffusion. Macromolecules. 2005;38:6128-6139.

33. Masubuchi Y, Takimoto J-I, Koyama K, Ianniruberto G, Marrucci G, Greco F. Brownian simulations of a network of reptating primitive chains. J of Chem Phys. 2001;115: 4387-4394.

34. Park SJ, Shanbhag S, Larson RG. A hierarchical algorithm for predicting the linear viscoelastic properties of polymer melts with long-chain branching. Rheologica Acta. 2005;44:319-330.

35. Wood-Adams PM, Dealy JM, deGroot AW, Redwine OD. Effect of molecular structure on the linear viscoelastic behavior of polyethylene. Macromolecules. 2000;33:7489-7499.

36. Costeux S, Wood-Adams PM, Beigzadeh D. Molecular structure of metallocene-catalyzed polyethylene: rheologically relevant representation of branching architecture in single catalyst and blended system. Macromolecules. 2002;35:2514-2528.

37. Das C, Inkson NJ, Read DJ, Kelmanson MA, McLeish TCB. Computational linear rheology of general branchon-branch polymers. $J$ of Rheology. 2006;50:207-235.

38. Mead DW, Larson RG, Doi M. A molecular theory for fast flows of entangled polymers. Macromolecules. 1998; 31:7895-7914.

39. Bent J, Hutchings LR, Richards RW, Gough T, Spares R, Coates PD, Grillo I, Harlen OG, Read DJ, Graham RS, Likhtman AE, Groves DJ, Nicholson TM, McLeish TCB. Neutron-mapping polymer flow: scattering, flow-visualization and molecular theory. Science. 2003;301:1691-1695.

40. McLeish TCB, Larson RG. Molecular constitutive equations for a class of branched polymers: the pom-pom polymers. J of Rheology. 1998;42:81-110. 